\title{
Wiring neurons with carbon nanotubes
}

\author{
William Lee and Vladimir Parpura*
}

Department of Neurobiology, Center for Glial Biology in Medicine, Atomic Force Microscopy \& Nanotechnology Laboratories, Civitan International Research Center, Evelyn F. McKnight Brain Institute, University of Alabama, Birmingham, USA

*Correspondence: vlad@uab.edu

\section{A commentary on}

Carbon nanotubes might improve neuronal performance by favouring electrical shortcuts.

by Cellot, G., Cilia, E., Cipollone, S., Rancic, V., Sucapane, A., Giordani, S., Gambazzi, L., Markram, H., Grandolfo, M., Scaini, D., Gelain, F., Casalis, L., Prato, M., Giugliano, M., and Ballerini, L. (2009). Nat. Nanotechnol. 4, 126-133.

Carbon nanotubes (CNTs) are emerging as nanomaterials with a wide range of biomedical applications, including their potential use for neuroprosthesis (Bekyarova et al., 2005). Thus, an understanding of how CNTs interact with cellular components of the brain is important for engineering of CNT-based devices. The conductive properties and nanoscale features of CNTs make them better suited as an interface with neurons for stimulating and recording neural activity than the conventional bare metal-based electrodes (Keefer et al., 2008). Neurons cultured on CNT substrates showed enhanced neuronal electrical activity (Lovat et al., 2005; Mazzatenta et al., 2007) suggesting an intimate interaction between neurons and CNTs. A recent study by Cellot et al. (2009) investigates the nature of CNT-neuron interactions and proposes a mechanism in which CNTs can boost neuronal activity by providing a shortcut for electrical coupling between somatic and dendritic neuronal compartments.

Cellot and colleagues performed wholecell patch clamp experiments on hippocampal and dorsal root ganglia (DRG) neurons cultured on planar CNT substrates deposited onto glass coverslips. They used a pharmacological approach to block neuronal network activity. Cultured neurons were bathed in 6-cyano-7-nitroquinoxaline-2,3-dione and gabazine to inhibit glutamatergic and gamma-amino-butyric acid-ergic synaptic transmission, respectively. This approach allowed for investigation of electrogenic properties of patch-clamped neurons since the endogenous, as opposed to input/synaptically driven, electrical excitability of cells remained intact. To study the contribution of CNTs to neuronal electrical regenerative properties, the whole-cell current-clamped single neurons were stimulated to discharge a train of six spikes/action potentials (APs), at frequencies ranging from 20 to $100 \mathrm{~Hz}$. The post-train changes in neuronal membrane potentials were recorded and analyzed. The integrated membrane potential changes occurring within a 100 -ms window starting $50 \mathrm{~ms}$ after the last AP of the train were categorized as: (a) after depolarization potential (ADP), (b) neutral response (NR), or (c) after hyperpolarization potential (AHP) (Table 1). Interestingly, hippocampal neurons grown on CNT-coated glass coverslips displayed a distribution of posttrain responses biased towards ADP when compared to the distribution obtained from neurons cultured on plain glass coverslips (Table 1).

After depolarization potential represents an electrical excitability based on dendro-somatic electrical coupling arising from summation of dendritic $\mathrm{Ca}^{2+}$

Table 1 |The type of membrane potential events, and their proportion, occurring as a follow-up to the train of action potentials evoked in hippocampal neurons grown on various substrates.

\begin{tabular}{|c|c|c|c|c|}
\hline \multirow[b]{2}{*}{ Substrate } & \multicolumn{3}{|c|}{ Post-train event } & \multirow[b]{2}{*}{ Source } \\
\hline & AHP & NR & ADP & \\
\hline \multicolumn{5}{|l|}{ GLASS } \\
\hline Neurons $(n)$ & 26 & 6 & 15 & Figure $1 b$ \\
\hline Proportion (\%) & 55 & 13 & 32 & \\
\hline \multicolumn{5}{|l|}{ CNTS } \\
\hline Neurons $(n)$ & 19 & 11 & 33 & Figure $1 b$ \\
\hline Proportion (\%) & 30 & 17 & 52 & \\
\hline \multicolumn{5}{|l|}{ ITO } \\
\hline Neurons $(n)$ & 19 & 2 & 6 & Figure $2 b$ \\
\hline Proportion (\%) & 70 & 7 & 22 & \\
\hline \multicolumn{5}{|l|}{ ITO+ } \\
\hline Neurons $(n)$ & 11 & 6 & 9 & Figure $2 b$ \\
\hline Proportion (\%) & 42 & 23 & 35 & \\
\hline \multicolumn{5}{|l|}{ ITO POOLED } \\
\hline Neurons $(n)$ & 30 & 8 & 15 & \\
\hline Proportion (\%) & 57 & 15 & 28 & \\
\hline
\end{tabular}

\section{RADA16}

Neurons $(n)$

Proportion (\%)

17

44
13

33
9

23

Listed figures from Cellot et al. (2009) in the utmost right hand column were used as a source for generating the number of events and their proportion from the whole. It should be noted that such an approach may lead to some of the minor discrepancies that exist between numbers reported in this table and those found in the text of the original article. The ITO pooled group represents the combined observations for low and high conductance substrates, denoted as ITO and ITO+, respectively.

ADP, after depolarization potential; AHP, after hyperpolarization potential; ITO, indium tin oxide; ITO+, high conductance ITO; NR, neutral response. 


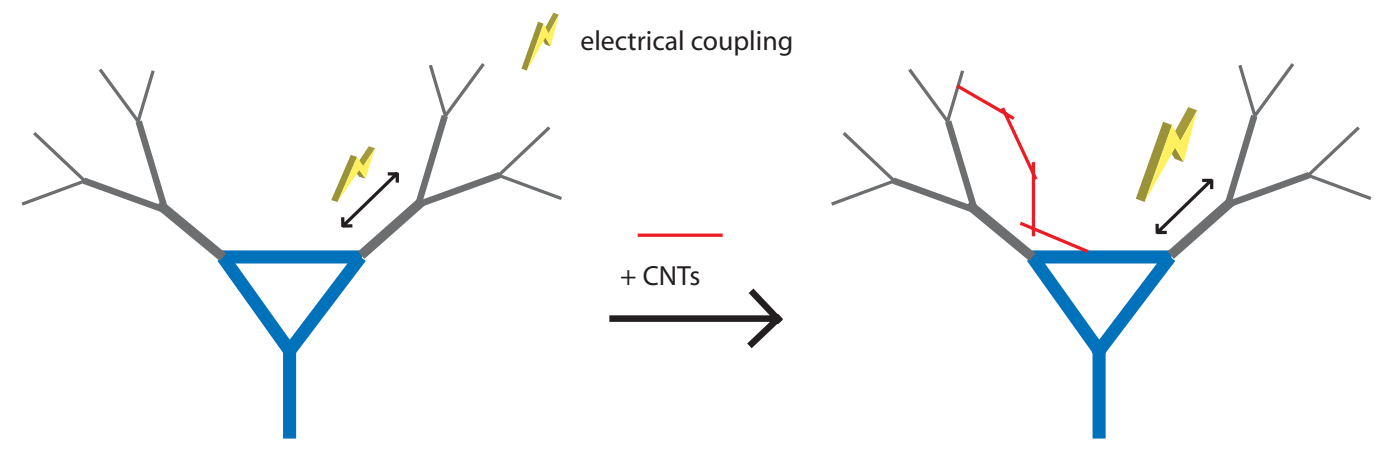

FIGURE 1 | Carbon nanotubes enhance excitability of hippocampal neurons by providing electrical shortcuts. (Left) Dendro-somatic electrical coupling (yellow bolt) arises from summation of dendritic $\mathrm{Ca}^{2+}$ influx triggered by back-propagating action potentials (double arrows). (Right) Carbon nanotubes (CNTs, red lines) can establish intimate contact with neurons causing enhancement of this electrical coupling influx triggered by back-propagating APs; pharmacological experiments revealed the ADP sensitivity to various voltage-gated $\mathrm{Ca}^{2+}$ channel blockers (Figure 1, left). It is then plausible that the CNT-induced change of neuronal excitability in the after math of trains of APs might be through the enhancement of dendro-somatic coupling (Figure 1, right). If so, then reducing the dendritic arborization in the neuron exposed to CNTs should result in the absence of ADPs. To assess this possibility, the authors performed the experiments using the same stimulation paradigm: a train of APs on DRG neurons. Unlike those from the hippocampus, DRG neurons contain next to no dendritic arborization. Following stimulation, DRG neurons did not exhibit any ADPs. The authors experimentally ruled out the alteration of intrinsic neuronal properties as a possible cause for the ADP enhancement by CNTs. Here hippocampal neurons were excited to discharge single APs, rather than a train of them. There was no appreciable difference in the amplitude or waveform of single APs recorded from neurons grown on CNT-coated and plain glass coverslips. Altogether, CNTs ability to enhance neuronal excitability after a discharge of a train of APs appears to be mediated by an enhanced dendro-somatic electrical coupling, a phenomenon carried out by $\mathrm{Ca}^{2+}$ influx via voltage-gated $\mathrm{Ca}^{2+}$ channels. It should be noted, however, that water-soluble CNTs can reduce depolarization-dependent $\mathrm{Ca}^{2+}$ influx from the extracellular space into hippocampal neurons (Ni et al., 2005). The fact that CNT substrates used to grow neurons did not block ADPs, but rather enhanced them, suggests that the presentation of CNTs (immobilized to a planar glass surface or watersoluble form), could dictate the type of effect exerted onto neurons. Additionally, this finding implies that substrates used have an excellent retention of CNTs immobilized to the glass coverslips, so that CNTs are not leaching out, which is consistent with findings elsewhere (Malarkey et al., 2009).

Carbon nanotube-coatings exhibit electrical conductivity and a nanoscale surface roughness, which is unlike a smooth and non-conductive glass surface. It has been demonstrated that a CNT substrate in a narrow range of conductivity can promote neuronal growth and neurite outgrowth (Malarkey et al., 2009). Although not systematically addressed, it has been implicated that surface roughness of CNTs can also affect neurite outgrowth (Sorkin et al., 2009). To address whether individual substrate qualities, conductivity or surface roughness, could play a role in mediating the observed effect of CNTs on neuronal excitability, Cellot et al. (2009) used two additional substrates to grow hippocampal neurons: (a) indium tin oxide (ITO), a substrate with electrical conductivity and a surface that lacks nanoscale roughness, and (b) RADA16 peptides, which can selforganize into nanofibers to form a substrate that is non-conductive, but displays a nanoscale surface roughness similar to that of CNT substrates. Pooled data from the examination of cultured neurons grown on ITO substrates showed that these cells exhibit electrical excitability that closely resembles that found in neurons grown on glass coverslips, rather than that of neurons grown on CNT substrates (Table 1). Here, two ITO substrates with different resistivity/conductivity, $5 \Omega \mathrm{mm} \quad(2 \mathrm{~S} / \mathrm{cm})$ and $0.005 \Omega \mathrm{mm}(2000 \mathrm{~S} / \mathrm{cm})$, were used. Hippocampal neurons grew on these ITO substrates similar as those cells plated onto plain glass, albeit neuronal morphological features in these conditions were not quantitatively assessed. Neurons were stimulated using the train of APs paradigm. It appeared that neurons grown on the ITO substrate with higher conductivity (ITO+) displayed more ADPs than those grown on ITO substrate with lower conductivity $(2 \mathrm{~S} / \mathrm{cm})$; the latter substrate conductivity is similar to the conductivity of CNT-coating deposited onto glass coverslips (resistivity of $1-1.2 \Omega \mathrm{mm}$, which corresponds to $\sim 9 \mathrm{~S} / \mathrm{cm}$ average conductivity). This phenomenon, although out of scope of the study, should be re-visited in the future and assessed by systematically varying conductive properties of CNTs while keeping their surface roughness unchanged. It may turn out that this could represent a tool for neuroprosthesis applications when modification of neuronal excitability would be desirable. Nonetheless, the proportion of ADPs recorded after the last AP from neurons grown on the ITO substrates were either similar to (ITO+), or lower than (ITO), the proportion recorded from neurons grown on glass coverslips (Table 1). Similarly, neurons cultured on RADA16 peptide substrates having a nanofiber meshwork similar to that formed by CNTs, but nonconductive, displayed a lower proportion of ADPs after a train of APs than neurons grown on smooth and non-conductive glass coverslips (Table 1). Additionally, a 
comparison of input resistance and capacitance measurements from neurons cultured on glass, CNT, ITO, and RADA16 peptides did not show any statistically significant difference between these different substrates which indicates that these intrinsic properties of neurons were unaltered. Thus, either the conductivity or the nanotexturing per se might not be responsible for the shift in the distribution of responses towards ADPs recorded in neurons grown on CNTs (Table 1). Such an effect might be specific to a substrate with the combination of electrical, chemical, and structural properties of CNTs.

As alluded to earlier, one possible explanation to the observation of ADP frequency shift is that the CNTs with their size and tubular shape as well as conductive characteristics could represent a shortcut for electrical coupling between two (somatic and dendritic) neuronal compartments. The authors substantiated this provocative hypothesis by theoretical modeling using a two-compartment spiking neuron model. They were, indeed, able to reproduce CNTmediated ADP and its sensitivity to $\mathrm{Ca}^{2+}$ channel blockers. They furthermore used modeling strategy to predict the effect of this CNT-mediated modulation of neuronal excitability on the neuronal networks. The prediction made based on computer simulations was then observed experimentally using electrophysiological recording from hippocampal neurons. Combined, these results indicate the existence of electrical coupling between CNTs and neurons. Such CNT-neuron interfaces should then be evident under electron microcopy (EM); the ultrastructural investigation using transmission EM showed tight contacts between CNTs and neuronal membranes. These hybrid areas should be of great interest for further investigations. One of the issues to address would be whether CNTs could induce $\mathrm{Ca}^{2+}$ "hot spots" via clustering of voltage-gated $\mathrm{Ca}^{2+}$ channels and/or their increased expression at the plasma membrane. Such action would represent an additional regulatory point for CNT modulation of the neuronal excitability. This would be especially interesting for neuroengineering, since it has been demonstrated that relatively low conductivity $(0.43-0.9 \mathrm{~S} / \mathrm{cm})$ woven polyester substrates enhanced the cell adhesion of endothelial cells and the level of interleukin- 6 mRNA in monocytes/macrophages (Jakubiec et al., 1998). Similar relatively low conductivity CNT substrates $(0.3 \mathrm{~S} / \mathrm{cm})$ caused neuronal morphological changes (Malarkey et al., 2009). Whether variations in conductivity of CNTs, while keeping their surface roughness relatively constant, can also correlate to changes in neuronal protein expression and excitability, and be harnessed for neuroengineering will need to be tested in carefully designed studies.

Taken together, Cellot et al. (2009) suggest that CNTs can establish intimate contacts with neurons resulting in the enhancement of neuronal electrical excitability. A proposed mechanism of action is that CNTs provide additional dendro-somatic electrical coupling pathways in parallel to the normal coupling route occurring through the neuronal cytosol/plasma membrane (Figure 1). While this model is the simplest explanation for neuronal enhancement by CNTs, the neuronal morphological changes induced by CNT substrates that could alter the passive neuronal membrane properties, as well as modifications of the level and spatio-temporal subcellular characteristics of protein expression, cannot be ruled out. The results from this study offer further support for the potential use of CNT-based electrical interfaces and scaffolds for guided regeneration of lost neurons at the site of brain injury.

\section{ACKNOWLEDGMENTS}

We thank Randy F. Stout Jr for his comments on a previous version of this commentary. The authors' work is supported by a grant from the National Institute of Mental Health (MH 069791 to VP). We dedicate this work to Glenn I. Hatton, whose energy and creativity inspired new views of astrocyte-neuronal interactions.

\section{REFERENCES}

Bekyarova, E., Ni, Y., Malarkey, E. B., Montana, V., McWilliams, J.L.,Haddon, R.C., and Parpura, V. (2005). Applications of carbon nanotubes in biotechnology and biomedicine. J. Biomed. Nanotech. 1, 3-17.

Cellot, G., Cilia, E., Cipollone, S., Rancic, V., Sucapane, A., Giordani, S., Gambazzi, L., Markram, H., Grandolfo, M., Scaini, D., Gelain, F., Casalis, L., Prato, M., Giugliano, M., and Ballerini, L. (2009). Carbon nanotubes might improve neuronal performance by favouring electrical shortcuts. Nat. Nanotechnol. 4, 126-133.

Jakubiec, B., Marois, Y., Zhang, Z., Roy, R., SigotLuizard, M. F., Dugre, F. J., King, M. W., Dao, L., Laroche, G., and Guidoin, R. (1998). In vitro cellular response to polypyrrole-coated woven polyester fabrics: Potential benefits of electrical conductivity. J. Biomed. Mater. Res. 41, 519-526.

Keefer, E. W., Botterman, B. R., Romero, M. I., Rossi, A. F., and Gross, G. W. (2008). Carbon nanotube coating improves neuronal recordings. Nat. Nanotechnol. 3 , 434-439.

Lovat, V., Pantarotto, D., Lagostena, L., Cacciari, B., Grandolfo, M., Righi, M., Spalluto, G., Prato, M., and Ballerini, L. (2005). Carbon nanotube substrates boost neuronal electrical signaling. Nano Lett. 5, 1107-1110.

Malarkey, E. B., Fisher, K. A., Bekyarova, E., Liu, W., Haddon, R. C., and Parpura, V. (2009). Conductive single-walled carbon nanotube substrates modulate neuronal growth. Nano Lett. 9, 264-268.

Mazzatenta, A., Giugliano, M., Campidelli, S., Gambazzi, L., Businaro, L., Markram, H., Prato, M., and Ballerini, L. (2007). Interfacing neurons with carbon nanotubes: electrical signal transfer and synaptic stimulation in cultured brain circuits. J. Neurosci. 27, 6931-6936.

Ni, Y., Hu, H., Malarkey, E. B., Zhao, B., Montana, V., Haddon, R. C., and Parpura, V. (2005). Chemically functionalized water soluble single-walled carbon nanotubes modulate neurite outgrowth. J. Nanosci. Nanotechnol. 5, 1707-1712.

Sorkin, R., Greenbaum, A., David-Pur, M., Anava, S., Ayali, A., Ben-Jacob, E., and Hanein, Y. (2009). Process entanglement as a neuronal anchorage mechanism to rough surfaces. Nanotechnology 20, 15101.

Received: 29 April 2009; published online: 29 May 2009. Citation: Lee W and Parpura V (2009) Wiring neurons with carbon nanotubes. Front. Neuroeng. 2:8. doi: 10.3389/neuro.16.008.2009

Copyright: () 2009 Lee and Parpura. This is an openaccess publication subject to an exclusive license agreement between the authors and the Frontiers Research Foundation, which permits unrestricted use, distribution, and reproduction in any medium, provided the original authors and source are credited. 